Doi: $10.32481 / \mathrm{djph} .2021 .12 .005$

\title{
Impact of Genetic Testing on Human Health:
}

\section{The Current Landscape and Future for Personalized Medicine}

Vicky L. Funanage, Ph.D.

Executive Director, Research, Nemours Children's Health

\begin{abstract}
Advances in the field of human genetics over the past three decades have led to improvements in human health through development and availability of novel genetic testing approaches for diagnosis, prognosis, treatment therapy, safety, preventive screening and population-based risk assessment. In this commentary, I highlight the current landscape of genetic testing in Delaware with a focus on the genetic etiology and molecular diagnosis of pediatric disease, tailored treatment efficacy and safety through novel clinical trials and pharmacogenomics, and training in the application of genomic approaches to rare and common diseases. Future opportunities include integrating application of genetic information into clinical practice, improving genetics education, focusing on disparities and access, and advancing genomics and digital health technologies.
\end{abstract}

Applied Clinical Genomics is the application of genetic information to the clinical setting, including improved diagnosis of disease and tailored treatment efficacy and safety. By discovering and defining the genes that underlie susceptibility to disorders, genetic information can be used to identify and better define those genes that play vital roles in human health, disease, and medical conditions. Equally important is the provision of training to physicians in the application of genomic and genetic approaches to studies of human diseases. When transferred directly into drug discovery programs and clinical trials, this knowledge will help to advance diagnosis, individualize treatment, and improve human health.

The mission of the Precision Medicine Program at Nemours Children's Health is to integrate the application of genetic information into patient care and to provide colleagues across our institution with the resources and knowledge base to adapt to the growing genetic and genomic landscape that impacts modern patient care. In 1993, the first Clinical Laboratory Improvements Amendments (CLIA)-certified pediatric genetic testing lab was established at the Alfred I. duPont campus in Delaware, with an initial focus on the neuromuscular disorders: Duchenne/Becker muscular dystrophy, myotonic dystrophy, Pelizaeus-Merzbacher disease, and spinal muscular atrophy. In addition to providing accurate diagnoses, the availability of these testing platforms led to the development of clinical decision support tools, improved clinical outcomes, and less invasive and expensive diagnostic tests that could replace muscle biopsy and histochemistry, which were the primary means for differential diagnosis of neuromuscular diseases in children. The genetic testing platforms also furthered understanding of the molecular etiology of myotonic dystrophy ${ }^{1,2}$ and Pelizaeus-Merzbacher disease ${ }^{3}$ and fostered the development of novel gene therapies for spinal muscular atrophy. ${ }^{4}$

While human genetics has long been known for its role in the diagnosis and management of familial genetic disorders such as cystic fibrosis and neurodevelopmental and neuromuscular disorders, variation in human DNA sequences contributes to risks for more common complex disorders including asthma, obesity, diabetes, autism, and congenital heart malformations. 
Response to treatment with medications and other procedures may also be largely determined by individual genetic variation. With rapidly advancing technologies in genome-wide mutation discovery (e.g., genome-wide association studies and next generation sequencing technologies), elucidation of the genomic risk factors or genetic etiology of complex diseases became possible. Moreover, "biomarker" profiles derived from many sources including patterns of gene activity, specific DNA sequences at important regulatory genes for drug metabolism (pharmacogenomics), or patterns of DNA sequences at other "risk" genes are rich sources of information for personalized approaches to medicine. These biomarker profiles, along with family history and educated practitioner assessments, can influence the management of patients in a positive way. The application of genetic information from bench to bedside, clinic to community, will help to improve diagnosis of disease, individualize treatment, and improve health outcomes.

Precision or Personalized Medicine, which considers individual differences in genes, environments and lifestyles to better understand and manage a patient's health, disease, or condition, has emerged as a paradigm shift in healthcare, with the intersection of big data and advances in genomics enabling efficient identification of high quality and personalized evidencebased care. The Nemours Precision Medicine Initiative was launched in 2017 to provide highquality, evidence-based care to children across the Nemours system, expanding the knowledge base for each individual patient to improve the overall safety, quality and cost effectiveness of their care. Several key programmatic pillars were included in this initiative. The Nemours Genetics Testing Stewardship Program was launched to provide consultation services for appropriate genetic testing, interpretation of genetic tests, explanation of test implications to caregivers and family, and diagnosis and management of genetic disorders. In a few short years, this program has been shown to increase access to genetic testing and provide prompt diagnoses and supportive care. ${ }^{5}$ The Nemours Pharmacogenomics Service Program, comprised of trained PharmD specialists, provides education, clinical decision support tools, and best practice alerts in the electronic health record (EHR). ${ }^{6,7}$ The Nemours Biomedical Research Informatics Center deployed a learning health system model, developing analytic tools to improve use of existing EHR data for outcomes research, and natural language processing and machine learning tools to make maximal use of unstructured medical data. ${ }^{8,9}$ The Biobank and Molecular Analysis Program offers a biorepository for storage of pediatric tissue samples as well as guidance and scientific support to investigators in the conduct of clinical research. Finally, the Rare Disease Program provides both single gene and next generation sequencing platforms to provide differential diagnoses for rare complex genetic disorders such as chronic kidney disease and leukodystrophies.

As healthcare organizations strive to excel in a value-driven healthcare economy, it will be important to accelerate the delivery of high quality, precise, and efficient care as well as provide cost savings for patients. Demonstration projects will incorporate genomic information into the EHR and provide clinical decision support for implementation of appropriate interventions or clinical advice. Dissemination of these methods through implementation science is a key goal so that the information generated will contribute to the growing knowledge base of using genomic information in patient care. Researchers often advance the practice of precision medicine by studying the genetics of a population, searching for commonalities and anomalies. This approach may unlock medical breakthroughs, enabling us to prevent disease, improve medication safety 
based on an individual's unique genetics, and better predict which treatments will be most effective.

Denny and Collins ${ }^{10}$ have proposed an expansion in seven key areas to enable a data-driven transformation in healthcare: large interoperable longitudinal cohorts, improved diversity and inclusion in science, big data and artificial intelligence, clinical genomics, phenomics, environment, privacy, trust and return of value. These areas provide a roadmap for precision medicine in 2030 and align with the overall goal of the National Academies' Roundtable on Genomics and Precision Health to ensure that all people benefit from genomics for precision health. ${ }^{11}$ The Genomics Workforce is prioritizing four action-oriented areas for 2021: innovation, dialog, equity, and adoption. Collectively, these areas of focus provide a roadmap for our state to employ its collective talent and voices to capitalize on the advances in genomics and precision health to improve the health of all Delawareans.

Correspondence: Dr. Funanage can be e-mailed at vicky.funanage@nemours.org

\section{References}

1. Carango, P., Noble, J. E., Marks, H. G., \& Funanage, V. L. (1993, November). Absence of myotonic dystrophy protein kinase $(D M P K)$ mRNA as a result of a triplet repeat expansion in myotonic dystrophy. Genomics, 18(2), 340-348. PubMed https://doi.org/10.1006/geno.1993.1474

2. Krahe, R., Ashizawa, T., Abbruzzese, C., Roeder, E., Carango, P., Giacanelli, M., .. . Siciliano, M. J. (1995, July 1). Effect of myotonic dystrophy trinucleotide repeat expansion on $D M P K$ transcription and processing. Genomics, 28(1), 1-14. PubMed https://doi.org/10.1006/geno.1995.1099

3. Taube, J. R., Sperle, K., Banser, L., Seeman, P., Cavan, B. C., Garbern, J. Y., \& Hobson, G. M. (2014, October 15). PMD patient mutations reveal a long-distance intronic interaction that regulates PLP1/DM20 alternative splicing. Human Molecular Genetics, 23(20), 54645478. Epub2014Jun1. PubMed

4. Finkel, R. S., Chiriboga, C. A., Vajsar, J., Day, J. W., Montes, J., De Vivo, D. C., .. . Farwell, W. (2021, July). Treatment of infantile-onset spinal muscular atrophy with nusinersen: Final report of a phase 2, open-label, multicentre, dose-escalation study. The Lancet. Child \& Adolescent Health, 5(7), 491-500. Epub2021Jun3. PubMed

5. Amlie-Wolf, L., Baker, L., Hiddemen, O., Thomas, M., Burke, C., Gluck, C., ... Gripp, K. W. (2021, April). Novel genetic testing model: A collaboration between genetic counselors and nephrology. American Journal of Medical Genetics. Part A, 185(4), 1142-1150. Epub2021Jan21. PubMed

6. Rahawi, S., Naik, H., Blake, K. V., Owusu Obeng, A., Wasserman, R. M., Seki, Y., .. . Scott, S. A. (2020, May). Knowledge and attitudes on pharmacogenetics among pediatricians. [published online ahead of print, 2020 Jan 27]. Journal of Human Genetics, 65(5), 437-444. PubMed https://doi.org/10.1038/s10038-020-0723-0

7. Cook, K. J., Duong, B. Q., Seligson, N. D., Arn, P., Funanage, V. L., Gripp, K. W., . . . Blake, K. V. (2021, September). Key considerations for selecting a genomic decision support platform for implementing pharmacogenomics. Clinical Pharmacology and 
Doi: $10.32481 /$ djph.2021.12.005

Therapeutics, 110(3), 555-558; Epub ahead of print. PubMed https://doi.org/10.1002/cpt.2328

8. Lang, J. E., Bunnell, H. T., Lima, J. J., Hossain, M. J., Wysocki, T., Bacharier, L., . . . Forrest, C. B. (2019, November). Effects of age, sex, race/ethnicity, and allergy status in obesity-related pediatric asthma. Pediatric Pulmonology, 54(11), 1684-1693. Epub2019Aug30. PubMed

9. Gupta, M., Bunnell, H. T., Phan, T. T., \& Beheshti, R. (2021, August). Concurrent Imputation and Prediction on EHR data using Bi-Directional GANs: Bi-GANs for EHR imputation and prediction. $A C M B C B, 2021,1$. PubMed

10. Denny, J. C., \& Collins, F. S. (2021, March 18). Precision medicine in 2030-seven ways to transform healthcare. Cell, 184(6), 1415-1419. PubMed https://doi.org/10.1016/j.cell.2021.01.015

11. Ginsburg, G., Penny, M., Feero, W. G., Miller, M., Addie, S., \& Beachy, S. H. (2021, October 7). The National Academies' Roundtable on Genomics and Precision Health: Where we have been and where we are heading. American Journal of Human Genetics, 108(10), 1817-1822. PubMed https://doi.org/10.1016/j.ajhg.2021.08.015

Copyright (c) 2021 Delaware Academy of Medicine / Delaware Public Health Association.

This is an Open Access article distributed under the terms of the Creative Commons Attribution Non-Commercial License (https://creativecommons.org/licenses/by-nc-nd/4.0/) which permits unrestricted non-commercial use, distribution, and reproduction in any medium, provided the original work is properly cited. 